University of Warwick institutional repository: http://go.warwick.ac.uk/wrap This paper is made available online in accordance with publisher policies. Please scroll down to view the document itself. Please refer to the repository record for this item and our policy information available from the repository home page for further information.

To see the final version of this paper please visit the publisher's website. Access to the published version may require a subscription.

Author(s): Sue Wharton

Article Title: Centre for Applied Linguistics at the University of Warwick

Year of publication: 2008

Link to published version:

http://dx.doi.org/10.1017/S0261444808005260

Publisher statement: None 


\section{Centre for Applied Linguistics at the University of Warwick}

The Centre for Applied Linguistics was established in 1983 and has grown from a relatively small teaching unit to a large centre engaged in a wide variety of activities under the broad heading of Applied Linguistics. Our work includes English language teaching, teacher education, undergraduate and postgraduate teaching in applied linguistics, development of teaching and research resources, and small- and large-scale research.

Research in the Centre focuses on the relationships between Applied Linguistics and professional practice in a range of international and multinational contexts. It benefits from some key resources such as the British Academic Spoken English and British Academic Written English corpora, and the ELT archive, a unique collection of historical ELT materials in a range of print and audio formats. It clusters around four main research areas: English Language Teacher Education and Development, Language Learning and Pedagogy, Professional and Academic Discourse, and Working and Communicating Across Cultures.

Work in English Language Teacher Education and Development emphasises above all the importance of critical reflection on situated practice. Research by the group works with teachers at a variety of career stages and a variety of professional contexts. Theoretical frameworks underpinning the research include individual perspectives such as teacher cognition, and social perspectives such as action research. They are unified, however, by a goal to encourage interaction with other professionals and engagement with theory and classroom research.

Work in Language Learning and Pedagogy also takes a multidisciplinary approach and emphasises the notion that learning and teaching are deeply embedded in particular contexts of practice. Focuses of research may be on any group of learners or teachers, but always from the perspective of appropriate pedagogy. Within this area also falls study of the field as a whole looking, for example, at the history of language teaching or at meta-activities such as materials analysis.

Research in Professional and Academic Discourse looks at text and discourse - both written and spoken - in a variety of professional and academic social contexts. We focus on the situated nature of text production and use, and therefore on the social antecedents and implications for people involved in various language events around the texts. Some of this research links directly with pedagogic research in the Centre. Our approaches to analysis include, for example, lexically-based computerised investigation of corpora, conversation analysis, genre analysis and systemic functional linguistics.

Research in Working and Communicating across Cultures investigates the impact of cultural factors on communication and interaction in a range of social, educational and professional contexts. We make use especially of work in pragmatics and discourse (e.g. face theory, 'politeness' theory, rapport management theory, multimodality) to examine the ways in which culture and language use interconnect. The research relates to language learning, teaching and teacher education in that we examine cultural conceptions of the role of the teacher, of 'good' student behaviour and 'good' teaching approaches, and that we look at intercultural effectiveness as a possible pedagogic goal. 
To give a flavour of some of the individual projects in which members of our centre are involved, we have chosen to concentrate on three doctoral projects, all close to completion. Each of these projects is reported in detail by the researcher responsible.

The first project falls mainly within the strand of Teacher Education and Development, and focuses on both teachers and classrooms. It is strongly rooted in a socially embedded view of teaching and learning, and also has a cross cultural dimension. The second project is also focused on classroom teaching, but this time from an action-research perspective in a university context. The third project concerns undergraduate learners in Bangladesh. It is largely based on the study of academic discourse, with a major focus on the analysis of student texts. In choosing to discuss these three projects in some detail, we aim to focus on the particular as much as on the general, in the hope that some readers will find points of resonance with their own work.

Sue Wharton

Centre for Applied Linguistics

University of Warwick

S.M.Wharton@warwick.ac.uk

\section{Teacher appraisal: The impact of an observer on teachers' classroom discourse}

The aim of this qualitative research project is to explore the influence of the presence of an observer on teacher methodology in classrooms where English is being taught as a foreign or second language (TESOL). The study attempts to identify any changes that the teachers may make in pedagogic practice in order to adapt to the presence of an observer, particularly if this person is there for appraisal purposes.

Most practising English language teachers are already familiar with observation as an appraisal tool. Not all English language teachers are observed, and there are alternative ways to carry out pedagogic appraisal; however, for teachers in some demographic areas and organisations, classroom appraisal by means of observation is a common experience. For those who do experience appraisal observations, the negative repercussions can sometimes be traumatic. This research was carried out in the Middle East, where unsuccessful observations can even result in compulsory relocation for the teacher's family.

For the purpose of this research, observed lessons are labelled 'model', and regular lessons are 'pedagogic'. The teacher participants (all native speakers of English) were asked to record themselves teaching both a model and a pedagogic lesson, so that the data could be transcribed. The resulting transcriptions are in the process of discourse analysis, and some interesting data have been identified. Theresa, a teacher in a tertiary college, provided recordings of a pair of lessons where the topic is weather. In the pedagogic lesson, there is an underlying rumble of student voices throughout the lesson segment, but the transcript indicates that they are working on the task, and are engaged in mutual discussion. Theresa's approach to the lesson seems to be conversational, but 
she uses word emphasis and frequent pauses to gain the students' attention. She also uses nomination as a device to correct student performance, and is able to spend time focussing on individual students. However, a clear structure to the lesson is not immediately obvious.

A sample from a corresponding model lesson provides some interesting contrasts. In this recording the ongoing rumble of student voices is missing, and a clear lesson structure is much more apparent. Turns clearly follow the Initiation-Response-Feedback sequence and there is a great deal of teacher control. Display questions are used extensively, and feedback is usually provided by echoing the students' responses. There is no nomination or correction, or use of stress to maintain attention, and teacher focus seems to be on the class rather than on individual students. As in the pedagogic lesson, pauses are still used for emphasis, but the silence between exchanges suggests that the students' attention is on their teacher at all times.

From these samples typical of Theresa's teaching, differences can already be identified in lesson structure, turn-taking, nomination, correction, and the way in which students and teacher relate to one another. It seems that she has made adjustments to her teaching style in order to accommodate the presence of an observer, and that the students may have made their own adaptations. Interestingly, analysis of transcripts from all the teachers suggests that the presence of any observer in the classroom may have a similar effect on both teacher and students, causing them to 'get the best china out', in the words of one of the Supervisor contributors. However, the observers identify personal preferences in terms of the elements that they are looking for in a model lesson, which suggests that it would not be true to say that the same format would work in all circumstances.

Amanda Howard

A.Howard@warwick.ac.uk

\section{EFL reading problems in a Mexican university: Proposing and assessing a potential solution}

This action-research project was conducted at the Universidad de Puebla (BUAP), in Mexico, where all the students learn English through four sequential and compulsory EFL modules which are taught communicatively but evaluated from a grammar-based perspective. This mismatch compels teachers to accomplish the 'programme' to have students 'ready for the exam'. In such a situation there is little opportunity to focus on the development of reading skills. This leads to difficulties for the university students, who face authentic English reading materials not for EFL course resources, but as a central source of updated information in their major academic disciplines. The objective of the project is to develop a reflective-didactic intervention to improve the reading situation.

Twenty-seven students in their final module participated in the study, which consists of two interconnected facets: the DESCRIPTIVE facet aims to assess the reading situation of the context before and after intervention. The DIDACTIC facet aims to bring about improvement to the 
teaching-learning situation. The connection between these two facets develops through three research stages.

1. Evaluative introduction stage - analysis of the existing reading status/situation before intervention. Main tools: (a) a reading test (first stage) that measures students' comprehension of an academic text, (b) an observation chart to trace subjects' reading, and (c) interviews/questionnaires.

2. Intervention/self-observation stage - to guide subjects towards improvement of their EFL reading by enhancing knowledge and practice of reading, of themselves as readers, and of their own reading. Main tools: (a) 14-session workshops, (b) a 'Reflexive Extensive Reading' programme to promote self-observation of subjects at reading, and (c) a diary.

3. Assessment stage - analysis of the way subjects approach EFL reading immediately after intervention. Main tools: (a) reading test (second stage) that measures students' comprehension of an academic text, and (b) interviews/questionnaires.

Preliminary test results indicate subjects' poor comprehension of academic texts before the intervention: the 18 subjects taking both first- and second-stage reading tests obtained only 174 points $(25.4 \%)$ out of 684 . Nevertheless, figures show improvement after intervention (199 points [27.1\%] out of 648). Although this improvement is not statistically significant, the tests allowed identification of the sections where subjects had more problems and the extent to which they coped with them. This information matches the qualitative information gathered from the other instruments. Such results point to the following trends: (i) identification of the major problem, i.e. limited vocabulary, and development of suitable strategy, i.e. guessing meaning through context, can be observed at stage 1 of the reading process; (ii) there is significant reliance on topic knowledge to generate specific ideas (micro-propositions); (iii) there is little incorporation of background knowledge as a whole. Learners seem to be aware that knowledge of the reading process and of personal weaknesses and strengths helps to develop suitable strategies for successful comprehension.

José Luís León Hernández

J.L.Hernandez@warwick.ac.uk

\section{A textual and contextual study of English language and literature essays: The case of first-year English department students' writing in Dhaka University, Bangladesh}

The stimulus for this study grew out of a concern for my students' poor performance in the writing of English essays and what could be done to improve the situation. I looked at English language and literature essays written by first-year students of the English department at Dhaka University (Bangladesh) from the perspective of genre analysis.

Since the main thrust of the study is text analysis, a total of 100 essays from the two contexts (language and literature) were analysed on the basis of Move-Strategy structure to see what 
structural patterns the essays possessed, what tactical choices students took to express the moves, and what was presented in terms of content matter within those moves. Two text analysis models (based on Exposition-Discussion and Recount-Description) were developed with move patterns based on previous studies following Swalesian genre tradition. In addition to text analysis, the study also conducted essay topic analysis - to investigate the type of topics students were required to write on - and interview and questionnaire surveys - to gain further contextual insights and specialist information from the members of the community.

Text analysis findings revealed that generically all essays possessed the three-part structure: Introduction, Body and Conclusion. The two models provided further insights into the MoveStrategy structure of these three main parts. The students were found to be writing essays belonging to three major classifications. For the language course mainly Recount-Description essays were practised with very few Exposition-Discussion essays, whereas only ExpositionDiscussion type analytical essays were written for literature courses. This indicated a mismatch between the type of writing that students needed in the main discipline and the type of writing taught in the adjunct course.

The Move-Strategy differed between the two models mainly in realisation of the moves in the Introduction and Body sections. The Exposition-Discussion essays were more complex structurally due to their analytical nature and complex content, whereas the RecountDescription essays were linear, anecdotal and often chronological.

Although the same model was used to analyse the Exposition-Discussion essays in both language and literature, there were differences in approach to content. Language essays, whether they were Recount-Description or Discussion-Explanation, were written from students' background knowledge, where subjectivity was essential and personal experience was treated as evidence. On the other hand, the writing of literature essays depended on multiple complex processes: reading of particular texts, understanding and synthesis of ideas with secondary sources, using discipline specialist language, engaging emotionally with the experiences in the text and then responding by transferring them into an organised and well argued written piece. For reasons of space only findings from 'Exposition-Discussion' will be presented here.

The most frequently occurring moves in the Introduction were Openers, realised by strategies like Gambit, Background Information, Definition and Proposition (P) realised by either Stating the Central Claim or Thesis. The Body of the essays consisted of two moves, Supporting Proposition (SP) and Elaborating Supporting Proposition (ESP), which reiterated $\mathrm{P}$ and elaborated SP with evidence and explanation, respectively. The conclusions were realised using the Restating Proposition move in literature essays, where there would be an affirmation of what preceded whereas the language essays were mainly realised with the Beyond Proposition move where students would offer suggestions and provide subjective evaluation.

The most problematic areas with the literature essays were in the relationship between Openers and Proposition in Introductions, and the realisation of the SP-ESP move pair in the Body paragraphs. Because literature essays were more critical and analytical, students were often too worried whether they should make their position explicit in the essay. For this reason, a stark contrast between the literature and language essay conclusions was noticed. In the literature essays, even the strongest students rarely took a stance and finished off their 
essays by restating what was written before; but in the language essays there was a deliberate drive to put forth their personal choices and recommendations.

These findings are put in perspective by the responses of the teachers and the students participating within the community. Students' difficulties in writing are attributed to the ways by which they were taught L1 and L2 essays, lack of instructions on structuring essays in the past, absence of instructions on literature essay writing at present, use of rote learning as a means to succeed in examinations, lack of critical reading ability, lack of opportunity to be creative, and writing under time constraints. These opinions, seen in the light of findings from text analysis, have pedagogical implications that can benefit students and teachers who would use genre-based approaches to teaching language and literature essay writing. Moreover, it can help university planners and policy makers to minimise the prevailing gap in the teaching-learning situation of students writing literature and language essays and develop the English curricula in an appropriate direction.

Nevin Farida

N.Farida@warwick.ac.uk 